\title{
"Ich will nicht als Nummer behandelt werden»
}

\section{Daniel Lüthi}

Freier Journalist, Fotograf, Medientrainer, Bern

Ein anderer würde krank an seiner Stelle. Mit allen möglichen und unmöglichen Diagnosen hat er sich $\mathrm{zu}$ identifizieren. So intensiv muss er sich mit physischen und psychischen Leiden auseinandersetzen und diese verkörpern, dass Fachleute denken, sie seien echt. Und trotzdem gilt es für ihn als Schauspieler gleichzeitig immer auch, die nötige Distanz zu wahren. "Nein, ein Hypochonder bin ich nicht, sonst könnte ich das nicht machen», sagt Roland Duppenthaler.

\section{Der eingebildete Kranke}

Seinen ersten Einsatz als "malade imaginaire», als eingebildeter Kranker, hatte er an der Fachhochschule für Gesundheit in Bern, an einer Prüfung für Physiotherapeuten. Eine Hüftarthrose und ein kaputtes Knie hatte er zu simulieren. Es kamen Auftritte bei Pflegenden und Ernährungsberaterinnen dazu, und dann auch regelmässige Einsätze bei Medizinstudentinnen und -studenten. An der Universität Bern wer-

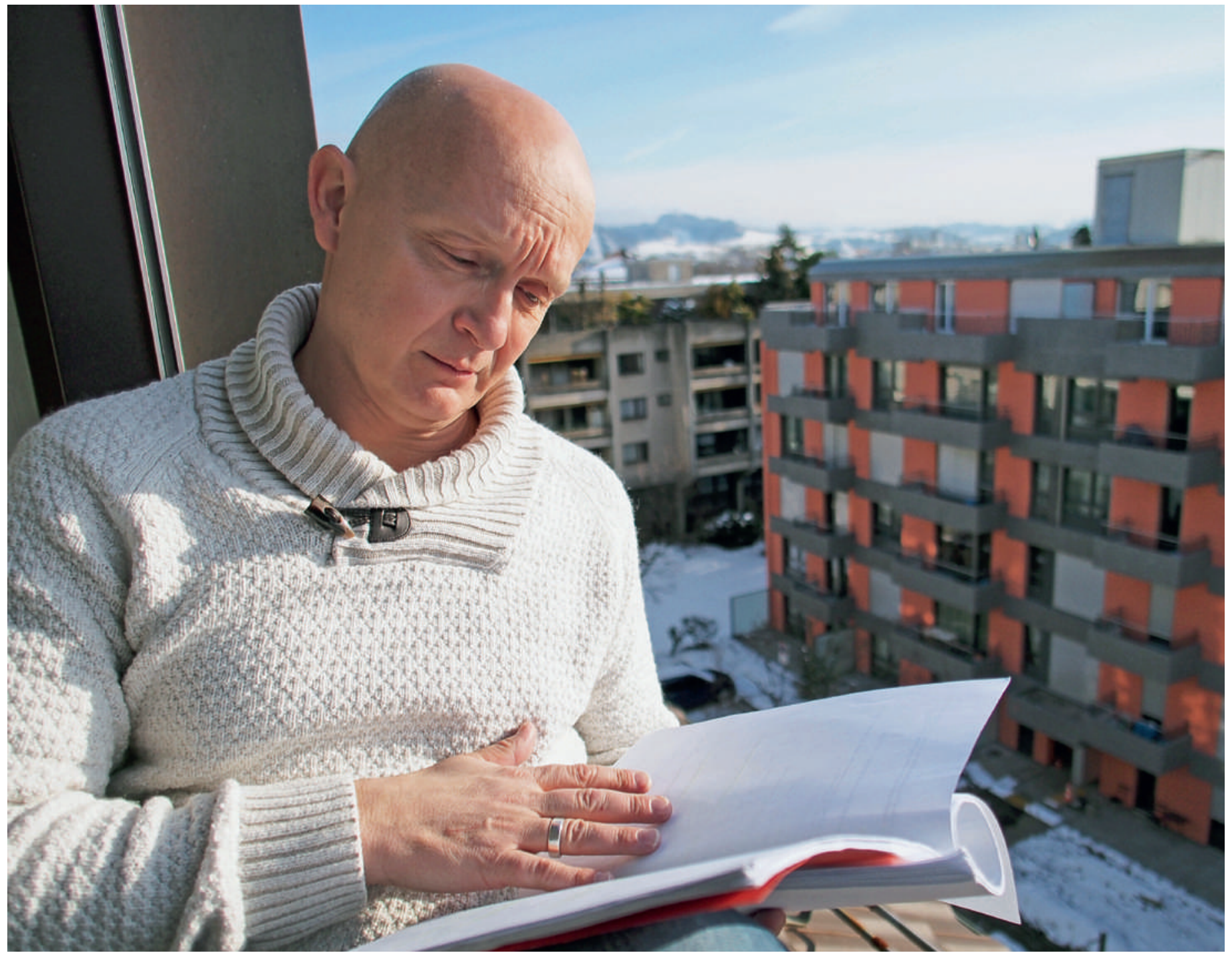


den sie vom Institut für medizinische Lehre organisiert. «Eine Sequenz an einer eidgenössischen Abschlussprüfung dauert nur zwischen acht und fünfzehn Minuten, dann kommt schon der oder die nächste.»

Die Fälle, mit denen dort die Studenten konfrontiert werden, sind von Fachleuten minutiös konstruiert, und sie werden zusammen mit dem Schauspieler gut vorbereitet. «Ich erhalte zu jedem Fall ein Skript mit einer Biografie und einem Krankheitsbild - und meiner Rolle. Zum Beispiel bin ich ein Patient mit Rückenschmerzen. Dafür muss ich wissen: Wo genau habe ich Schmerzen? Welche Qualität haben diese Schmerzen? Und wie sind meine Bewegungsmuster?» Gerade bei solchen körperlichen Symptomen sei es schon vorgekommen, erzählt Roland Duppenthaler, dass der imaginäre Schmerz zu einem realen Problem geworden sei: «Wenn ich die gleiche Fehlhaltung über 30mal nacheinander praktiziert habe, kann es schon vorkommen, dass ich am Ende des Tages wirkliche Schmerzen verspüre.»

\section{Ein standardisierter Patient}

Eben: Duppenthaler ist ja kein Patient, sondern ein Schauspiel-Patient. Beziehungsweise, wie es im Fachjargon heisst, ein standardisierter Patient. «Es geht darum, dass drei verschiedene Schauspieler den gleichen Fall gleich spielen können, so dass verschiedene Studenten an verschiedenen Universitäten möglichst die gleiche Ausgangslage und die gleichen Chancen haben. Konkret heisst das beispielsweise, dass ich immer mit dem gleichen Satz beginne.» Bis 36mal pro Tag kann dies der Fall sein. Wird das nicht langweilig für einen professionellen Schauspieler? Die Wiederholung gehöre überall dazu, antwortet Duppenthaler, auch auf einer grossen Bühne. Aber auch sonst sei diese Arbeit für ihn keineswegs eine Schauspielerei zweiten Ranges: «Es ist eine grosse Herausforderung, immer wieder von neuem möglichst realistisch zu sein. Und immer wieder auf den gleichen Level zu kommen. Im Übrigen steht für mich meine Selbstverwirklichung als Schauspieler hier nicht im Vordergrund. Entscheidend ist, dass die anderen, die Übenden und Prüflinge, möglichst viel profitieren. Ich sehe mich als Material für die Studenten.»

\section{Kommunikative Kompetenzen}

Ob ein Fall aus der Geriatrie, der Orthopädie oder der Inneren Medizin: Es geht darum, dass der Student die richtigen Fragen stellt und die richtigen Tests macht.

\section{Roland Duppenthaler}

Roland Duppenthaler wurde 1966 in Bern geboren. Er wuchs in Bolligen auf. In Bern besuchte er das Lehrerseminar. Nach Erlangung des Primarlehrerpatents wollte er Schauspieler werden. Am Theater "Die Katakombe» in Frankfurt erhielt er sein erstes Engagement, dann war er in verschiedenen Produktionen in Deutschland und

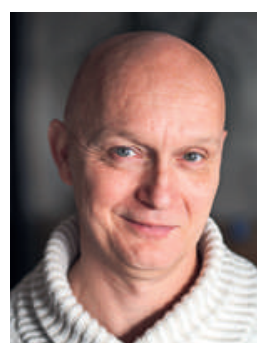

der Schweiz tätig, so auch am damaligen Städtebundtheater Biel-Solothurn. Seit 2006 arbeitet er als Schauspiel-Patient in verschiedenen Schweizer Städten und an verschiedenen Institutionen, so an den medizinischen Fakultäten der Universitäten Bern und Zürich. Roland Duppenthaler lebt mit seinem Partner in Bern.

Es ist also sicher eines der Hauptziele eines Trainings oder einer Prüfung, Fachkenntnisse abzurufen. Vom Fachgebiet her muss der Schauspieler absolut flexibel sein: «Das geht von einer Verkrümmung der Nasenscheidenwand bis zu einer Krebsdiagnose, also möglicherweise bis zu einem Todesurteil.» Und als Patient habe er bisweilen verstockt und renitent zu sein, «manchmal aber weiss ich auch alles besser und sprudle wie ein Wasserfall.» Will heissen: Es geht nicht nur um fachtechnische Fähigkeiten, sondern auch um kommunikative Kompetenzen. «Wenn immer möglich, wird auch das therapeutische Klima bewertet.» Als Schauspiel-Patient könne er dazu bei Kommunikationstrainings jeweils auch eine Bewertung abgeben. «Das Spektrum der Reaktionen ist sehr breit. Einige Studenten tun so, als ob sie schon alles wüssten.» Manchmal verspüre er eine grosse Abneigung oder

\section{"Ich sehe mich als Material für die Studenten.»}

Wut und merke, dass er sich von dieser Person im richtigen Leben nie und nimmer behandeln lassen möchte. «Rühr mich bloss nicht an und stell ja nicht noch eine blöde Frage mehr», denke er dann zum Beispiel. «Insgesamt aber habe ich den Eindruck, dass Kommunikation in der Medizin zu einem wichtigen Thema geworden ist, dass Studentinnen und Studenten sensibilisiert sind dafür, zuhören können und auch bereit sind, kritische Rückmeldungen anzunehmen.» Wenn immer möglich, versuche er, sein Gegenüber von der Chance einer solchen Übung zu überzeugen: «Möglicherweise ist es das einzige Mal, dass ein Patient ein ehrliches Feedback gibt.»

\section{Fiktion und Wirklichkeit}

A propos Spektrum: «Einen betrunkenen oder einen psychotischen Patienten spiele ich schon lieber als 
einen kaputten Ellbogen», gesteht Roland Duppenthaler, der schauspielerische Handlungsspielraum sei bei psychischen Krankheiten vielleicht doch grösser als bei einfachen körperlichen Einschränkungen. Die Szenen spielen sich bisweilen in einem SprechstundenSetting ab, manchmal liegt der Schauspiel-Patient aber auch in einem Spitalbett. Zunehmend werden die Sequenzen mit Video aufgezeichnet. «Wie stark ich jeweils in die Emotion gehe, ist offen. Ich muss und kann nicht 36-mal nacheinander weinen. Aber bei einer besonders empathischen Studentin sind mir auch schon die Tränen gekommen.»

\section{"Insgesamt habe ich den Eindruck, dass Kommunikation in der Medizin zu einem wichtigen Thema geworden ist.»}

Es kommt vor, dass Duppenthaler einen Angehörigen zu spielen hat, "einen werdenden Vater bei einer Hebamme oder einen Ehemann, dem man mitteilt, dass seine Frau im Sterben liegt.» Aber als Angehöriger hat Roland Duppenthaler auch seine ganz realen persönlichen Erfahrungen gemacht. Sein Partner habe vor einiger Zeit eine Hirnblutung erlitten, erzählt er, jetzt spürbar betroffen, und auf seiner Odyssee durch verschiedene Spitäler sei er beim Empfang als Erstes immer gefragt worden: "Wie ist er versichert?» Getröstet habe ihn dann die Erfahrung, dass er in der Intensiv-Station des Inselspitals mit Respekt und Empathie empfangen worden sei.

Das sei etwas, was er auch als Schauspiel-Patient merke: «Ich will nicht als Nummer behandelt werden, sondern als Mensch. Ich darf doch erwarten, dass ich nicht ‘Zimmer 3 rechts` bin, sondern bei meinem Namen genannt werde. Und dass es zur Professionalität eines Arztes gehört, dass er auf seine Patienten eingeht, und nicht einfach davon ausgeht, die seien selber schuld an ihrem Schicksal.» Wichtig in diesem Zusammenhang sei das Bewusstsein, das Sensorium. «Dazu tragen unserer Trainings Entscheidendes bei.» Selber war Duppenthaler auch einmal Patient in einem Spital, und der Zufall wollte es, dass er die dortige Ernährungsberaterin zuvor einmal als Schauspiel-
Patient erlebt hatte: «Wir hatten das Heu überhaupt nicht auf der gleichen Bühne, was unsere Begegnung im realen Spitalalltag natürlich schwierig machte.» So können sich Fiktion und Wirklichkeit ganz plötzlich vermischen.

\section{Grosse Gesten und kleiner Kummer}

Wenn Roland Duppenthaler nicht gerade einen Patienten mimt, ist er oft mit der Produktion «Dinnerkrimi» auf Tournee oder spielt auch in Musicals. Eine seiner Lieblingsrollen war die des Conférenciers im Musical «Cabaret». «Die Kombination von Schauspiel, Gesang und Tanz entspricht mir am meisten.» Hier kann er sich schauspielerisch auch mehr ausleben als in der Patientenrolle.

Das Spektrum seiner möglichen Einsätze auf einer grossen Bühne ist wegen eines kleinen körperlichen Mangels eingeschränkt. Duppenthaler vermittelt das Detail mit einer Mischung aus Erstaunen, Unverständnis und Belustigung. Es ist eine alte Geschichte, mit der er längst zu leben gelernt hat. Eine aber auch, die typisch ist für die Welt eines Schauspielers, der sich ständig zwischen grossen Gesten und kleinem Kummer, zwischen Wirklichkeit und Imagination bewegt. Als Kind habe er den rechten Arm gebrochen, erzählt Roland Duppenthaler, und der sei nicht richtig zusammengewachsen. "Jetzt habe ich einen krummen Arm. Deswegen wurde ich vor 25 Jahren an einer Musicalschule in Deutschland nicht aufgenommen und sehe damit manchmal in einem Musical-Ensemble in der Chorus-Line nicht gut aus.»

Eine Geschichte, zu der ein anderer Satz des Schauspiel-Patienten besonders gut passt: «Wer das macht, darf nicht eitel sein.»

\section{Die nächste «Begegnung mit ...»}

Am Ende jeden Monats stellt die Schweizerische Ärztezeitung eine Persönlichkeit vor, die sich im Gesundheitswesen engagiert. Im Mai schildert Daniel Lüthi seine Begegnung mit Andreas Meyer-Heim, ärztlicher Leiter Rehabilitation am Universitäts-Kinderspital Zürich 